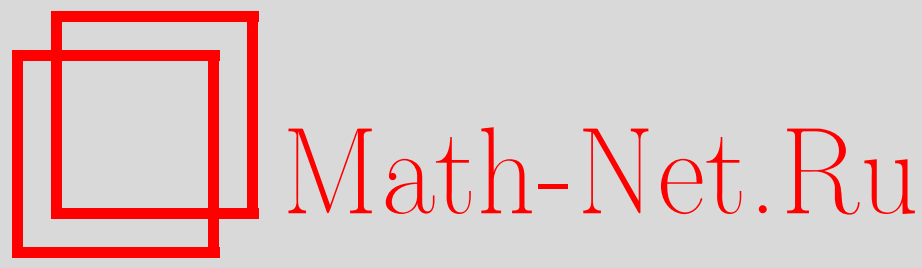

А. А. Хамзин, Р. Р. Нигматуллин, Термодинамика модели равных спин-спиновых взаимодействий, ТМФ, 2010, том 165, номер 1, 160-176

DOI: https://doi.org/10.4213/tmf6569

Использование Общероссийского математического портала Math-Net.Ru подразумевает, что вы прочитали и согласны с пользовательским соглашением http: //www . mathnet.ru/rus/agreement

Параметры загрузки:

IP : 54.80 .97 .219

26 апреля 2023 г., 09:14:29

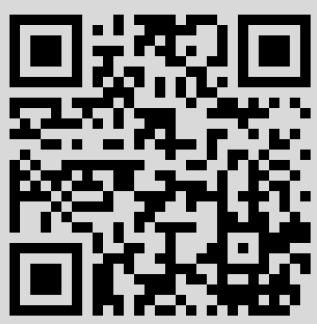




\title{
ФИЗИКА
}

Том 165, № 1

октябрь, 2010

2010 г.

\author{
А. А. Хамзин ${ }^{* \dagger}$, Р. Р. Нигматуллин ${ }^{\dagger}$
}

\section{ТЕРМОДИНАМИКА МОДЕЛИ РАВНЫХ СПИН-СПИНОВЫХ ВЗАИМОДЕЙСТВИЙ}

\begin{abstract}
Рассмотрена термодинамика модели равных спин-спиновых взаимодействий. Получены точные выражения для корреляционных функций и теплоемкости конечных кластеров, справедливые во всем интервале температур и внешних полей. Проведен анализ полученных термодинамических характеристик в зависимости от параметров взаимодействия, внешнего магнитного поля и числа частиц в кластере. Обнаружено аномальное поведение теплоемкости и других термодинамических величин, обусловленное возникновением в спектре элементарных возбуждений спиновой щели. Отсутствие дальнего порядка в системе обусловливается присутствием в системе топологических возбуждений (солитонов). Изучено влияние параметров анизотропного взаимодействия на структуру солитонов.
\end{abstract}

Ключевые слова: спин-спиновое взаимодействие, термодинамика, спиновый кластер, корреляционная функция, теплоемкость.

\section{1. ВВЕДЕНИЕ}

В работе [1] была предложена упрощенная модель описания свойств спиновых систем в мезоскопических магнитоактивных кластерах. Эти кластеры могут быть использованы в качестве рабочих сред для реализации алгоритмов квантовых компьютеров [2],[3], области физики, бурно развивающейся в последнее десятилетие. Эта модель равных спин-спиновых взаимодействий (РССВ) определяется гамильтонианом

$$
\begin{aligned}
H & =-h \sum_{f} S_{f}^{z}-\frac{A}{2} \sum_{f, f^{\prime}} S_{f}^{z} S_{f^{\prime}}^{z}-\frac{B}{4} \sum_{f, f^{\prime}}\left(S_{f}^{+} S_{f^{\prime}}^{-}+S_{f^{\prime}}^{-} S_{f}^{+}\right)= \\
& =-h S^{z}-\frac{1}{2}(A-B)\left(S^{z}\right)^{2}+\frac{N}{8}(A+2 B)-\frac{B}{2} S^{2},
\end{aligned}
$$

*Казанский государственный энергетический университет, Казань, Россия. E-mail: airat.khamzin@rambler.ru

${ }^{\dagger}$ Казанский (Приволжский) федеральный университет, Казань, Россия. E-mail: nigmat@knet.ru 
где $A$ и $B$ - средние значения соответственно продольных и поперечных компонент реального взаимодействия между спинами, $h$ - внешнее магнитное поле,

$$
S^{z}=\sum_{f=1}^{N} S_{f}^{z}, \quad S^{ \pm}=\sum_{f=1}^{N} S_{f}^{ \pm}
$$

- компоненты полного спина $\vec{S}, N$ - число частиц в кластере, $S^{2}$ - квадрат полного спина, который определяется выражением

$$
S^{2}=\frac{S^{+} S^{-}+S^{-} S^{+}}{2}+\left(S^{z}\right)^{2}
$$

Изотропный вариант модели $(A=B)$ исследовался в работе [4], в которой обнаружена неэргодичность динамики данной модели.

В настоящей работе мы продолжим начатое в статье [1] изучение модели РССВ и рассмотрим термодинамическое поведение спинов, связанных равными взаимодействиями. Анализ термодинамики любой многочастичной системы является неотъемлемой частью полноценного изучения модели, претендующей на описание физических свойств реальных систем.

\section{2. ТОЧНЫЙ РАСЧЕТ ТЕРМОДИНАМИЧЕСКИХ ХАРАКТЕРИСТИК МОДЕЛИ РССВ ДЛЯ КОНЕЧНЫХ КЛАСТЕРОВ}

Для расчета термодинамических характеристик рассматриваемой модели необходимо знание корреляционных функций $R_{k l}=\left\langle\left(S^{z}\right)^{k-1}\left(S^{2}\right)^{l-1}\right\rangle$, где $k=1,2, \ldots, N+1$, $l=1,2, \ldots,[N / 2]+1$, поскольку операторы $S^{z}$ и $S^{2}$ в случае частиц со спином $1 / 2$ удовлетворяют соотношениям

$$
\begin{aligned}
\left(S^{z}\right)^{N+1} & =\sum_{k=1}^{N+1} p_{k}\left(S^{z}\right)^{k-1}, \\
\left(S^{2}\right)^{[N / 2]+1} & =\sum_{l=1}^{[N / 2]+1} q_{l}\left(S^{2}\right)^{l-1} .
\end{aligned}
$$

Коэффициенты $p_{k}$ и $q_{l}$ легко находятся из уравнений $r_{1}\left(S^{z}\right)=0, r_{2}\left(S^{2}\right)=0$, где $r_{1}(\lambda)$ и $r_{2}(\lambda)$ - минимальные аннулирующие многочлены для диагональной матрицы $S^{z}$ и матрицы $S^{2}$ соответственно, которые определяются выражениями

$$
r_{1}(\lambda)=\prod_{m=-N / 2}^{N / 2}(\lambda-m), \quad r_{2}(\lambda)=\prod_{s=s_{\min }}^{N / 2}(\lambda-s(s+1)),
$$

где $s_{\min }=1 / 2$ для нечетного количества спинов $N$ и $s_{\min }=0$ для четного количества спинов $N$ в кластере.

Корреляционные функции $R_{k l}$ могут быть легко рассчитаны непосредственно, поскольку гамильтониан (1) имеет простой вид и его собственные значения равны

$$
E_{m s}=-h m-\frac{B}{2}(\xi-1) m^{2}-\frac{B}{2} s(s+1)+\frac{N B}{8}(\xi+2),
$$

6 Теоретическая и математическая физика, т. 165, № 1, 2010 г. 
где $s=s_{\min }, \ldots, N / 2, m=-s, \ldots, s, \xi=A / B$, причем спектр гамильтониана является вырожденным, и степень вырождения каждого уровня равна

$$
g(N, s)=\frac{2 s+1}{N+1}\left(\begin{array}{c}
N+1 \\
s+1+N / 2
\end{array}\right)
$$

где $\left(\begin{array}{c}N+1 \\ s+1+N / 2\end{array}\right)$ - биномиальный коэффициент. Действительно,

$$
R_{k l}=\left\langle\left(S^{z}\right)^{k-1}\left(S^{2}\right)^{l-1}\right\rangle=\frac{\operatorname{Sp}\left(\left(S^{z}\right)^{k-1}\left(S^{2}\right)^{l-1} e^{-\beta H}\right)}{\operatorname{Sp}\left(e^{-\beta H}\right)} .
$$

Подставляя сюда собственные значения (5), получаем

$$
R_{k l}=\frac{\sum_{s=s_{\min }}^{N / 2} \sum_{m=-s}^{s} g(N, s) m^{k-1}(s(s+1))^{l-1} e^{\beta h m+(\beta B / 2)\left[(\xi-1) m^{2}+s(s+1)\right]}}{\sum_{s=s_{\min }}^{N / 2} \sum_{m=-s}^{s} g(N, s) e^{\beta h m+(\beta B / 2)\left[(\xi-1) m^{2}+s(s+1)\right]}} .
$$

Отсюда видно, что в нулевом магнитном поле $(h=0) R_{k 1}=0$ при четном значении $k$. Таким образом, в конечных кластерах спонтанная намагниченность не возникает $\left(k=2,\left\langle S^{z}\right\rangle=0\right)$. В приложении можно найти другой подход к расчету корреляционных функций $R_{k l}$.

Средняя удельная энергия системы выражается через функции $R_{21}, R_{31}, R_{12}$ и имеет вид

$$
\frac{1}{N B}\langle H\rangle=-\frac{h}{N B} R_{21}-\frac{\xi-1}{2 N} R_{31}-\frac{1}{2 N} R_{12}+\frac{\xi+2}{8} .
$$

Из этой формулы легко получить выражение для удельной теплоемкости модели в нулевом магнитном поле:

$$
\frac{1}{N B} C_{V}=\frac{1}{N B} \frac{d\langle H\rangle}{d T}=\frac{1}{2 N k_{\mathrm{B}} T^{2}}\left((\xi-1) \frac{d R_{31}}{d \beta}+\frac{d R_{12}}{d \beta}\right) .
$$

Подставляя сюда выражения для функций $R_{31}$ и $R_{12}$,

$$
\begin{aligned}
R_{31} & =\frac{\sum_{s=s_{\min }}^{N / 2} \sum_{m=-s}^{s} g(N, s) m^{2} e^{\beta h m+(\beta B / 2)\left[(\xi-1) m^{2}+s(s+1)\right]}}{\sum_{s=s_{\min }}^{N / 2} \sum_{m=-s}^{s} g(N, s) e^{\beta h m+(\beta B / 2)\left[(\xi-1) m^{2}+s(s+1)\right]}}, \\
R_{12} & =\frac{\sum_{s=s_{\min }}^{N / 2} \sum_{m=-s}^{s} g(N, s)(s(s+1)) e^{\beta h m+(\beta B / 2)\left[(\xi-1) m^{2}+s(s+1)\right]}}{\sum_{s=s_{\text {min }}}^{N / 2} \sum_{m=-s}^{s} g(N, s) e^{\beta h m+(\beta B / 2)\left[(\xi-1) m^{2}+s(s+1)\right]}},
\end{aligned}
$$

получим

$$
\frac{1}{N k_{\mathrm{B}}} C_{V}=\frac{\beta^{2}}{N} \frac{d^{2}}{d \beta^{2}} \ln \left(\sum_{s=s_{\min }}^{N / 2} g(N, s) \sum_{m=-s}^{s} \exp \left(\frac{\beta}{2}\left[(\xi-1) m^{2}+s(s+1)\right]+\beta h^{\prime} m\right)\right) .
$$

Здесь и далее будем считать, что $\beta=B / k_{\mathrm{B}} T, h^{\prime}=h / B, B>0$.

Зная корреляционные функции $R_{k l}$, легко найти выражения для продольных и поперечных двухчастичных, а также многочастичных корреляционных функций. Действительно, имеем

$$
R_{31}=\left\langle\left(S^{z}\right)^{2}\right\rangle=\sum_{f, g=1}^{N}\left\langle S_{f}^{z} S_{g}^{z}\right\rangle=\frac{N}{4}+\sum_{f \neq g=1}^{N}\left\langle S_{f}^{z} S_{g}^{z}\right\rangle=\frac{N}{4}+N(N-1)\left\langle S_{f}^{z} S_{g}^{z}\right\rangle,
$$


поскольку каждый спин одинаково взаимодействует с остальными. Отсюда непосредственно находим искомое выражение для продольной двухчастичной корреляционной функции:

$$
\left\langle S_{f}^{z} S_{g}^{z}\right\rangle=\frac{1}{N-1}\left(\frac{R_{31}}{N}-\frac{1}{4}\right) .
$$

Поперечная двухчастичная корреляционная функция $\left\langle S_{f}^{+} S_{g}^{-}\right\rangle$также может быть легко найдена из равенства $S^{2}=S^{+} S^{-}-S^{z}+\left(S^{z}\right)^{2}$. Действительно,

$$
\begin{aligned}
\left\langle S^{+} S^{-}\right\rangle & =\sum_{f, g=1}^{N}\left\langle S_{f}^{+} S_{g}^{-}\right\rangle=\sum_{f=1}\left(\frac{1}{2}+\left\langle S_{f}^{z}\right\rangle\right)+\sum_{f \neq g=1}^{N}\left\langle S_{f}^{+} S_{g}^{-}\right\rangle= \\
& =\frac{N}{2}+N(N-1)\left\langle S_{f}^{+} S_{g}^{-}\right\rangle=R_{12}-R_{31},
\end{aligned}
$$

откуда легко находим

$$
\left\langle S_{f}^{+} S_{g}^{-}\right\rangle=\frac{1}{N-1}\left(\frac{R_{12}-R_{31}}{N}-\frac{1}{2}\right) .
$$

Многочастичные корреляционные функции $\left\langle S_{f_{1}}^{z} S_{f_{2}}^{z} \ldots S_{f_{l}}^{z}\right\rangle$ аналогично выражаются через корреляционные функции $R_{k 1}$, но более высокого порядка. Так, например, четырехчастичный коррелятор имеет вид

$$
\left\langle S_{f_{1}}^{z} S_{f_{2}}^{z} S_{f_{3}}^{z} S_{f_{4}}^{z}\right\rangle=\left(\begin{array}{c}
N \\
4
\end{array}\right)^{-1}\left(R_{51}-\frac{3 N}{2} R_{31}\right) .
$$

\section{3. ОБСУЖДЕНИЕ РЕЗУЛЬТАТОВ ПРОВЕДЕННЫХ РАСЧЕТОВ}

На рис. 1-4 представлены графики зависимостей удельной теплоемкости, продольной и поперечной двухчастичных корреляционных функций от температуры при различных значениях параметра $\xi$ и числа частиц в кластере в нулевом магнитном поле. Видно, что поведение теплоемкости и корреляционных функций как функций от температуры существенным образом определяется числом частиц в кластере и параметром взаимодействия $\xi=A / B$. Так, при значениях параметра $\xi \neq 1$ наблюдается аномальное поведение теплоемкости при низких температурах (см. рис. 1 и 2), возникает дополнительный к основному максимум, который отчетливо проявляется при малых значениях $\xi-1>0$ и относительно небольших значениях числа спинов в кластере $N$. Аналогичное аномальное поведение в низкотемпературном поведении теплоемкости выявлено для конечных гейзенберговских спиновых цепочек, имеющих топологию “гребенки" при малых значениях взаимодействия в "зубьях" по сравнению с взаимодействием в основной цепочке [5], а также в фрустрированных системах Изинга [6]. Данное аномальное поведение теплоемкости характерно для систем со спиновой щелью в спектре элементарных возбуждений. Получим двухпиковую структуру температурной зависимости теплоемкости аналитически, исследуя ее точное выражение (7) при низких температурах.

Согласно формуле (7) удельная теплоемкость выражается через статистическую сумму, которая без несущественного множителя $e^{-\beta B(\xi+2) / 8}$ имеет вид

$$
Z=\sum_{s=s_{\min }}^{N / 2} g(N, s) \sum_{m=-s}^{s} \exp \left\{\frac{\beta}{2}\left(m^{2} \delta+s(s+1)\right)+h^{\prime} \beta m\right\},
$$




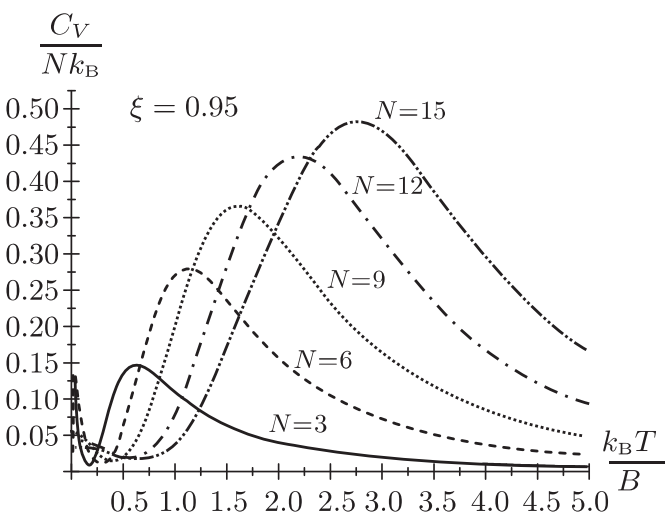

a

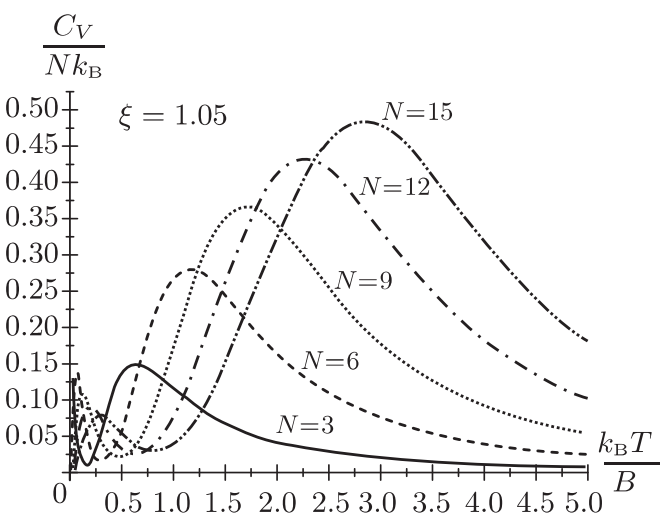

б

Рис. 1. Зависимость удельной теплоемкости модели РССВ от температуры для различных значений числа спинов $N$ при $\xi=0.95$ (а) и $\xi=1.05$ (б).

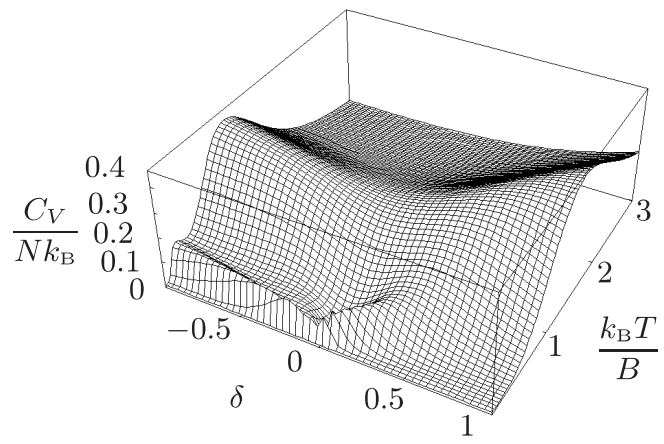

Рис. 2. Зависимость удельной теплоемкости модели РССВ от температуры и параметра анизотропии $\delta=A / B-1$ в нулевом магнитном поле при $N=6$.

где $\delta=\xi-1$. В соответствии с неравенством Чебышева статистическую сумму (8) в нулевом магнитном поле представим в виде $Z \geqslant Z_{1} Z_{2}$, где

$$
\begin{aligned}
& Z_{1}=\frac{2}{N+2-2 s_{\min }} \sum_{s=s_{\min }}^{N / 2} \sum_{m=-s}^{s} \exp \left(\frac{\beta}{2} m^{2} \delta\right) \approx 2 \sum_{m=s_{\min }}^{N / 2} \exp \left(\frac{\beta}{2} m^{2} \delta\right), \\
& Z_{2}=\sum_{s=s_{\min }}^{N / 2} g(N, s) \exp \left(\frac{\beta}{2} s(s+1)\right) .
\end{aligned}
$$

Тогда удельная теплоемкость записывается как сумма двух слагаемых:

$$
\frac{1}{N k_{\mathrm{B}}} C_{V}=\frac{\beta^{2}}{N} \frac{d^{2}}{d \beta^{2}} \ln Z=c_{1}+c_{2},
$$

где

$$
c_{1,2}=\frac{\beta^{2}}{N} \frac{d^{2}}{d \beta^{2}} \ln Z_{1,2}
$$




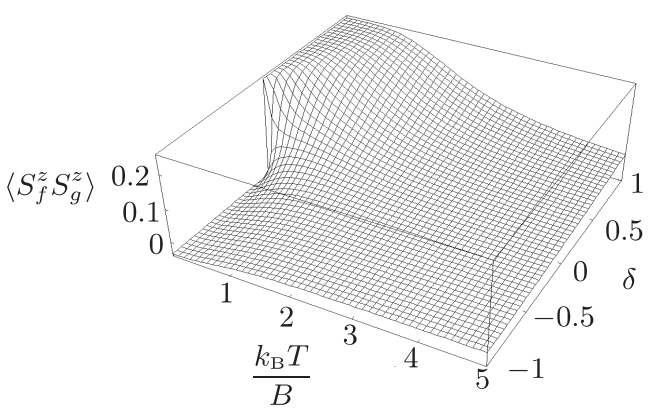

a

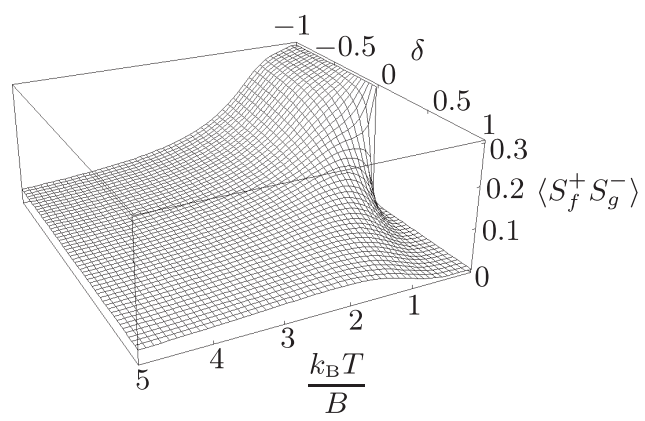

6

Рис. 3. Зависимости продольной $\left\langle S_{f}^{z} S_{g}^{z}\right\rangle$ (а) и поперечной $\left\langle S_{f}^{+} S_{g}^{-}\right\rangle$(б) двухчастичных корреляционных функций от температуры и параметра анизотропии $\delta=A / B-1$ в нулевом магнитном поле при $N=6$.

При низких температурах в статистической сумме $Z_{2}$ удержим два первых лидирующих слагаемых, тогда

$$
\begin{aligned}
Z_{2} & \approx g\left(N, \frac{N}{2}\right) \exp \left(\frac{\beta}{4} N\left(\frac{N}{2}+1\right)\right)+g\left(N, \frac{N}{2}-1\right) \exp \left(\frac{\beta}{4} N\left(\frac{N}{2}-1\right)\right)= \\
& =\exp \left(\frac{\beta}{4} N\left(\frac{N}{2}+1\right)\right)\left(1+(N-1) \exp \left(-\frac{\beta}{2} N\right)\right) .
\end{aligned}
$$

В результате

$$
\begin{aligned}
c_{2} & \approx \frac{\beta^{2}}{N} \frac{d^{2}}{d \beta^{2}}\left(\frac{\beta N}{4}\left(\frac{N}{2}+1\right)+\ln \left(1+(N-1) \exp \left(-\frac{\beta}{2} N\right)\right)\right) \approx \\
& \approx \frac{\beta^{2}}{N} \frac{d^{2}}{d \beta^{2}}\left(\frac{\beta N}{4}\left(\frac{N}{2}+1\right)+(N-1) \exp \left(-\frac{\beta}{2} N\right)\right)=\frac{N(N-1)}{4} \beta^{2} \exp \left(-\frac{\beta N}{2}\right) .
\end{aligned}
$$

Теплоемкость $c_{2}$ имеет максимум порядка $4 / e^{2}$ при температуре $T_{m 2} \simeq N / 4$, что согласуется с результатами численных расчетов, представленными на рис. 1 и 2 .

Найдем выражения для первого слагаемого в теплоемкости. Вначале рассмотрим случай $\delta>0$ (анизотропия типа "легкая ось"). Тогда, снова удерживая в сумме два первых лидирующих члена, представим статистическую сумму $Z_{1}$ в виде

$$
Z_{1} \approx 2 \exp \left(\frac{\beta}{8} N^{2} \delta\right)\left(1+\exp \left(-\frac{\beta \delta}{2}(N-1)\right)\right) .
$$

В результате вклад $c_{1}$ в теплоемкость запишется как

$$
\begin{aligned}
c_{1} & \approx \frac{\beta^{2}}{N} \frac{d^{2}}{d \beta^{2}}\left(\ln 2+\frac{N^{2} \beta \delta}{8}+\ln \left(1+\exp \left(-\frac{\beta \delta}{2}(N-1)\right)\right)\right) \approx \\
& \approx \frac{\beta^{2} \delta^{2}(N-1)}{4} \exp \left(-\frac{\beta \delta(N-1)}{2}\right) .
\end{aligned}
$$

Слагаемое $c_{1}$ определяет аномальное поведение теплоемкости при низких температурах, которое связано с появлением в спектре элементарных возбуждений спиновой 


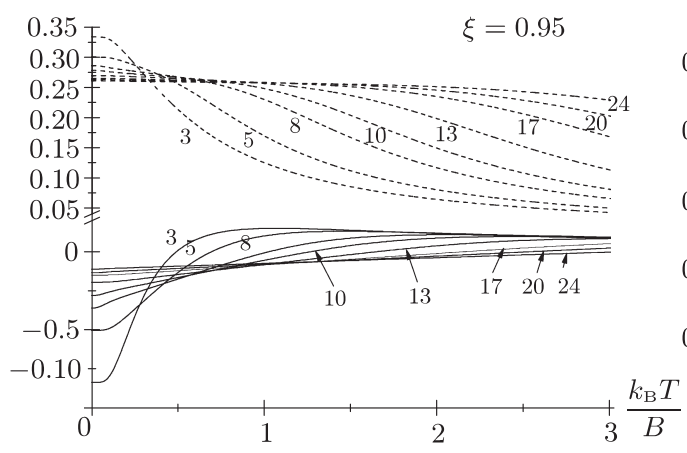

a

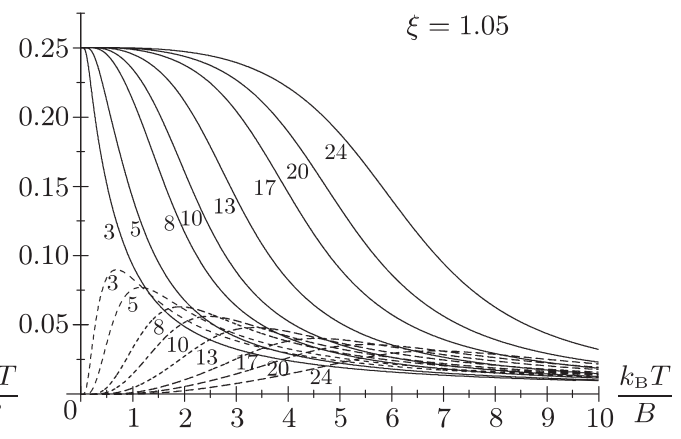

б

Рис. 4. Зависимости двухчастичных корреляционных функций $\left\langle S_{f}^{z} S_{g}^{z}\right\rangle$ (сплошные кривые) и $\left\langle S_{f}^{+} S_{g}^{-}\right\rangle$(штриховые кривые) от температуры при различных значениях числа частиц в кластере $(N=3,5,8,10,13,17,20,24)$ для $\xi=0.95$ (а) и $\xi=1.05$ (б).

щели. Величина щели, как можно видеть из (10), равна $\Delta \approx \delta(N-1) / 2$. В термодинамическом равновесии вероятность рождения щелевого возбуждения пропорциональна $e^{-\beta \Delta}$, а число возбуждений равно $Q=N e^{-\beta \delta(N-1) / 2}$. Условие $Q>1$ выполняется при определенном соотношении между числом частиц в кластере и температурой:

$$
T>\frac{N \delta}{2 \ln N}=T^{*} .
$$

Теплоемкость $c_{1}$ имеет максимум порядка $4 / N e^{2}$ при температуре $T_{m 1} \simeq N \delta / 4$, что согласуется с результатами численных расчетов, представленными на рис. 1 и 2 .

Аномальное поведение теплоемкости при понижении температуры проявляется начиная с некоторой температуры $T_{0}$, соответствующей локальному минимуму зависимости теплоемкости от температуры (см. рис. 1)

$$
T_{0} \approx \frac{N(1-\delta)}{2 \ln \left(N / \delta^{2}\right)}
$$

Таким образом, с учетом (11) аномальное поведение теплоемкости проявляется в интервале температур

$$
\Delta T=T_{0}-T^{*}=\frac{N}{2}\left(\frac{1-\delta}{\ln \left(N / \delta^{2}\right)}-\frac{\delta}{\ln N}\right),
$$

поэтому спиновая щель существует при

$$
0<\delta<\delta_{0}=-\frac{\ln N}{2 W(-\ln N /(2 N))} \approx \frac{1}{2}\left(1-\frac{1}{\log _{2} N}\right)
$$

где $W(x)$ - функция Ламберта, определяемая соотношением $W e^{W}=x$.

Случай $\delta<0$ (анизотропия типа "легкая плоскость") существенно отличается. В этом случае в статистической сумме $Z_{1}$ первые два лидирующих слагаемых имеют 
иной вид:

$$
Z_{1} \approx 2 \exp \left(-\frac{\beta|\delta| s_{\min }^{2}}{2}\right)\left(1+\exp \left(-\frac{\beta|\delta|\left(2 s_{\min }+1\right)}{2}\right)\right)
$$

поэтому

$$
\begin{aligned}
c_{1} & \approx \frac{\beta^{2}}{N} \frac{d^{2}}{d \beta^{2}}\left(\ln 2-\frac{s_{\min }^{2} \beta|\delta|}{2}+\ln \left(1+\exp \left(-\frac{\beta|\delta|\left(2 s_{\min }+1\right)}{2}\right)\right)\right) \approx \\
& \approx \frac{\beta^{2} \delta^{2}\left(2 s_{\min }+1\right)^{2}}{4 N} \exp \left(-\frac{\beta|\delta|\left(2 s_{\min }+1\right)}{2}\right) .
\end{aligned}
$$

Таким образом, в случае $\delta<0$ величина щели в спектре элементарных возбуждений меньше, чем в случае $\delta>0$ и равна $\Delta^{\prime}=|\delta|\left(2 s_{\min }+1\right) / 2$. Температура, выше которой число щелевых возбуждений $Q^{\prime}=N e^{-\beta \Delta^{\prime}}>1$, равна

$$
T^{* \prime}=\frac{|\delta|\left(2 s_{\min }+1\right)}{2 \ln N},
$$

а температуры максимума аномальной составляющей теплоемкости и локального минимума соответственно равны

$$
T_{m 1}^{\prime} \approx \frac{|\delta|\left(2 s_{\min }+1\right)}{4}<T_{m 1}, \quad T_{0}^{\prime} \approx \frac{N-\left(2 s_{\min }\right)|\delta|}{2 \ln \frac{N^{2}(N-1)}{\delta^{2}\left(2 s_{\min }+1\right)^{2}}}<T_{0}
$$

Интервал значений параметра $\delta$, при которых открывается щель и проявляется аномальное поведение теплоемкости, значительно шире:

$$
0<|\delta|<\delta_{0}^{\prime}=-\frac{N \ln N}{2\left(2 s_{\min }+1\right) W(-\ln N /(2 N))} \approx \frac{N}{2\left(2 s_{\min }+1\right)}\left(1-\frac{1}{\log _{2} N}\right) .
$$

В работах [7]-[9] теоретически и экспериментально показано, что аномальная температурная зависимость теплоемкости и других величин связана с наличием в системе топологических солитонов. Ниже в рамках классического рассмотрения будет показано, что в рассматриваемой системе действительно присутствуют топологические возбуждения (солитоны), которые обусловливают отсутствие дальнего порядка в рассматриваемой модели и формируют указанное специфическое поведение теплоемкости.

В соответствии с классическим рассмотрением в гамильтониане (1) заменим спиновые операторы компонентами классического вектора длины $S=1 / 2$ :

$$
S_{f}^{x}=\frac{1}{2} \sin \theta_{f} \cos \varphi_{f}, \quad S_{f}^{y}=\frac{1}{2} \sin \theta_{f} \sin \varphi_{f}, \quad S_{f}^{z}=\frac{1}{2} \cos \theta_{f} .
$$

В результате для рассматриваемой модели энергия может быть представлена как функционал

$$
E\{\theta, \varphi\}=\frac{B}{4} \sum_{f<g}\left(1-\xi \cos \theta_{f} \cos \theta_{g}-\sin \theta_{f} \sin \theta_{g} \cos \left(\varphi_{f}-\varphi_{g}\right)\right) .
$$


Поскольку взаимодействие спинов однородно в плоскости $x O y$, рассмотрим случай $\varphi_{f}=$ const. Распределение $\theta_{f}$ минимизирует функционал $E\{\theta\}$ и удовлетворяет уравнению $\delta E / \delta \theta_{f}=0$, которое для модели (12) записывается в виде

$$
\xi \sin \theta_{f} \sum_{g} \cos \theta_{g}=\cos \theta_{f} \sum_{g} \sin \theta_{g} .
$$

Решить данное уравнение в общем случае не представляется возможным, поэтому рассмотрим его в непрерывном пределе. Полагая, что $\theta_{f}$ меняется достаточно слабо от точки к точке, с точностью до производных второго порядка по пространственным координатам запишем уравнение (12) в виде

$$
\left(1+\varepsilon \delta \sin ^{2} \theta\right) \Delta \theta=\varepsilon \delta\left(\frac{d}{R^{2}}-\frac{1}{2} \nabla \theta^{2}\right) \sin 2 \theta,
$$

где $d$ - размерность пространства, $R$ - расстояние между спинами, параметр $\varepsilon$ определяет анизотропию типа “легкая ось" $(\varepsilon=1)$ и “легкая плоскость" $(\varepsilon=-1)$, а $\delta>0$.

Так как в рассматриваемой модели каждый спин одинаково взаимодействует со всеми остальными спинами, целесообразно геометрически представить пространственное расположение спинов, взаимодействующих с выделенным, на сфере некоторого радиуса $R$. Тогда уравнение (14) в сферических координатах $(r=R, \vartheta, \phi)$ перепишется в виде

$$
\begin{aligned}
\left(1+\varepsilon \delta \sin ^{2} \theta\right) & \left(\frac{1}{\sin \vartheta} \frac{\partial}{\partial \vartheta}\left(\sin \vartheta \frac{\partial \theta}{\partial \vartheta}\right)+\frac{1}{\sin ^{2} \vartheta} \frac{\partial^{2} \theta}{\partial \phi^{2}}\right)= \\
= & \varepsilon \delta\left(d-\frac{1}{2}\left(\left(\frac{\partial \theta}{\partial \vartheta}\right)^{2}+\frac{1}{\sin ^{2} \vartheta}\left(\frac{\partial \theta}{\partial \phi}\right)^{2}\right)\right) \sin 2 \theta
\end{aligned}
$$

Снова в общем случае решить полученное уравнение не представляется возможным, поэтому рассмотрим случай $\theta=\theta(\vartheta)$, тогда уравнение (15) становится обыкновенным дифференциальным уравнением

$$
\left(1+\varepsilon \delta \sin ^{2} \theta\right) \frac{1}{\sin \vartheta} \frac{\partial}{\partial \vartheta}\left(\sin \vartheta \frac{\partial \theta}{\partial \vartheta}\right)=\varepsilon \delta\left(d-\frac{1}{2}\left(\frac{\partial \theta}{\partial \vartheta}\right)^{2}\right) \sin 2 \theta
$$

Введем новую переменную $\eta=\ln \operatorname{tg}(\vartheta / 2)$, тогда уравнение (16) примет вид

$$
\left(1+\varepsilon \delta \sin ^{2} \theta\right) \theta_{\eta \eta}^{\prime \prime}=\varepsilon \delta\left(d \sin ^{2} \vartheta-\frac{1}{2}\left(\theta_{\eta}^{\prime}\right)^{2}\right) \sin 2 \theta .
$$

Решение будем искать в виде $\cos \theta=u$. В результате уравнение (17) для новой функции $u$ может быть записано как

$$
\left(1+\varepsilon \delta\left(1-u^{2}\right)\right) u^{\prime \prime}+\frac{u}{1-u^{2}}\left(u^{\prime}\right)^{2}=-d \delta \varepsilon u\left(1-u^{2}\right)
$$

где для упрощения дальнейшего анализа мы заменили $\sin ^{2} \vartheta$ его средним значением $1 / 2$. Уравнение (18) поддается решению, и вид зависимости $\theta(\eta)$ в квадратурах таков:

$$
\int_{\theta\left(\eta_{0}\right)}^{\theta(\eta)} \frac{\sqrt{1+\delta \varepsilon \sin ^{2} \theta}}{\sqrt{c-d \delta \varepsilon \cos ^{2} \theta}} d \theta=\eta-\eta_{0}
$$

где $c, \eta_{0}$ - постоянные. 
Рассмотрим сначала случай анизотропии типа “легкая ось" $(\varepsilon=1)$. Как было показано выше, аномальное поведение теплоемкости существенно проявляется при слабой анизотропии спин-спиновых взаимодействий $(0<\delta \ll 1)$, поэтому в данном случае уравнение $(18)$ примет более простой вид $\left(u^{\prime}\right)^{2} \approx d \delta\left(1-u^{2}\right)^{2}$. Его решение есть

$$
\cos \theta= \pm \operatorname{th}\left(\sqrt{d \delta} \ln \operatorname{tg} \frac{\vartheta}{2}\right)
$$

Оно описывает вклад топологических солитонов типа доменных стенок, энергия которых в рамках непрерывного подхода равна

$$
E_{\mathrm{s}}=2 \pi R^{2} \frac{1}{4} B \int_{0}^{\pi} \sin \vartheta\left(\frac{R^{2}(1+\delta) \nabla \theta^{2}-2 d \delta \cos ^{2} \theta}{1+\delta \sin ^{2} \theta}\right) d \vartheta \approx \pi B R^{2} \sqrt{d \delta} .
$$

Из этой формулы видно, что энергия солитонов отлична от нуля при $\delta \neq 0$, поэтому солитоны возникают и дают вклад в термодинамику при отличной от нуля анизотропии. Ширина доменной стенки обратно пропорциональна $\sqrt{\delta}$.

В работах [10]-[12] исследовались ферромагнетики со слабой анизотропией типа “легкая ось" для гейзенберговского гамильтониана; показано, что в спиновых системах образуются спиновые комплексы - связанные состояния $N$-магнонов, где энергия магнитной анизотропии соответствует парному притяжению длинноволновых магнонов. Связанные состояния магнонов (“магнонная капля") фактически являются зародышем области с обратным направлением намагниченности в безграничном однодоменном магнетике. В рассматриваемой модели, по-видимому, мы имеем дело с образованием подобных спиновых комплексов (солитонов), которые и определяют специфическую термодинамическую картину.

На рис. 5 показано распределение профиля намагниченности $m_{z}=(1 / 2) \cos \theta$ на поверхности сферического кластера единичного радиуса при различных значениях параметра анизотропии $\delta$.

Рассмотрим случай с анизотропией типа "легкая плоскость" $(\varepsilon=-1)$. При произвольных значениях $\delta$ решение $\theta(\eta)$ описывается уравнением (19), это решение мы представим в виде

$$
\Pi\left(\psi,-\frac{\delta}{1-\delta}, \frac{k^{2}-\delta}{1-\delta}\right)=\frac{\sqrt{d \delta(1-\delta)}}{k}\left(\eta-\eta_{0}\right),
$$

где П $(\psi, \nu, p)$ - эллиптический интеграл третьего рода,

$$
k^{2}=\frac{d \delta}{c+d \delta}, \quad \psi=\arcsin \left(\sqrt{1-\delta} \frac{\sin \theta}{\sqrt{1-\delta \sin ^{2} \theta}}\right) .
$$

Нахождение явной зависимости $\theta(\eta)$ связано с проблемой обращения эллиптического интеграла третьего рода. Решение (21) описывает периодическое в $\eta$-пространстве распределение намагниченности около нуля: $-k \leqslant \cos \theta \leqslant k$. Период колебаний намагниченности равен

$$
T(k, \delta)=4 \pi k \int_{0}^{\pi / 2} \frac{\sqrt{1-\delta \sin ^{2} \theta}}{\sqrt{1+k^{2} \cos ^{2} \theta}} d \theta
$$




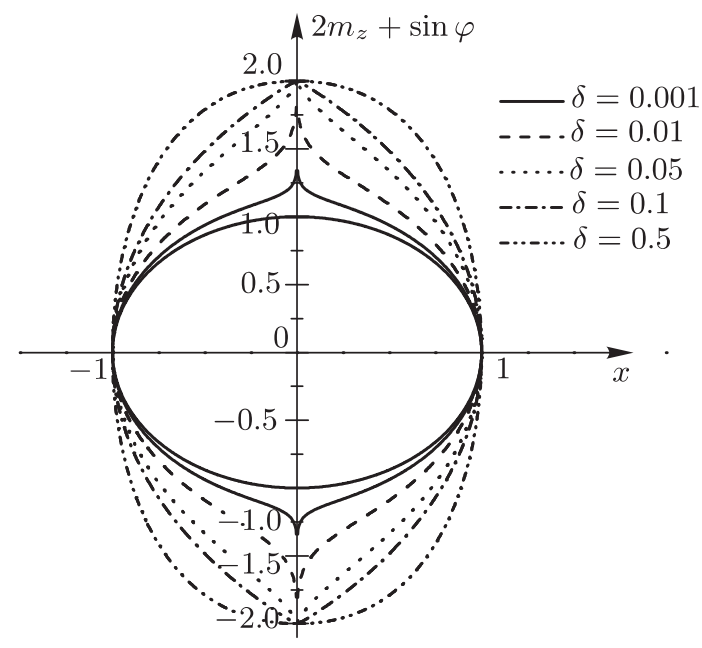

Рис. 5. Профили намагниченности $m_{z}(\vartheta)$ при различных значениях параметра анизотропии $\delta=A / B-1$ на поверхности сферического кластера единичного радиуса.

и является монотонно возрастающей функцией как параметра $k$, так и параметра анизотропии $\delta$. Решению (21) соответствует система концентрических (по переменной $\eta$ ) на поверхности сферы кольцевых доменов.

При $\delta=-1$ ( $X Y$-модель) уравнение (18) имеет решение $\sin \theta=\cos \left(\sqrt{d}\left(\eta-\eta_{0}\right)\right)$. Распределение профиля намагниченности на поверхности сферы для $X Y$-модели изображено на рис. 6. Видно, что на поверхности сферы образуются концентрические домены. Качественно такая картина распределения намагниченности остается и при произвольных значениях $\delta$. Энергия солитонов в этом частном случае легко вычисляется по формуле $(12)$ и равна $E_{\mathrm{s}}=2 d \pi R^{2} B$.

Поведение продольной и поперечной двухчастичных корреляционных функций при низких температурах имеет следующий вид: при четных $N$

$$
\begin{aligned}
\left\langle S_{f}^{z} S_{g}^{z}\right\rangle & =\frac{1}{N-1}\left(\frac{2}{N \beta} \frac{d}{d \delta} \ln Z_{1}-\frac{1}{4}\right) \approx \\
& \approx \begin{cases}\frac{1}{N-1}\left(-\frac{1}{4}+\frac{1}{N} e^{-\beta|\delta| / 2}+\cdots\right), & \delta<0, \\
\frac{1}{4}-\frac{1}{N} e^{-\beta \delta(N-1) / 2}+\cdots, & \delta>0,\end{cases} \\
\left\langle S_{f}^{+} S_{g}^{-}\right\rangle & \approx \frac{1}{N-1}\left(\frac{2}{N}\left(\frac{d}{d \beta} \ln Z_{2}-\frac{1}{\beta} \frac{d}{d \delta} \ln Z_{1}\right)-\frac{1}{2}\right) \approx \\
& \approx \begin{cases}\frac{1}{N-1}\left(\frac{N}{4}-\frac{1}{N} e^{-\beta|\delta| / 2}+\cdots\right), & \delta<0, \\
\frac{1}{N} e^{-\beta \delta(N-1) / 2}+\cdots, & \delta>0 ;\end{cases}
\end{aligned}
$$




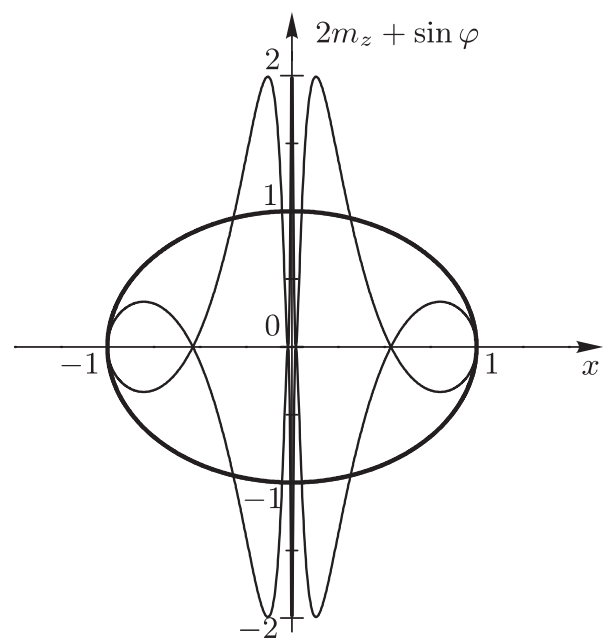

Рис. 6. Профили намагниченности $m_{z}(\vartheta)$ для $X Y$-модели на поверхности сферического кластера единичного радиуса.

при нечетных $N$

$$
\begin{aligned}
\left\langle S_{f}^{z} S_{g}^{z}\right\rangle & =\frac{1}{N-1}\left(\frac{2}{N \beta} \frac{d}{d \delta} \ln Z_{1}-\frac{1}{4}\right) \approx \\
& \approx \begin{cases}\frac{1}{N}\left(-\frac{1}{4}+\frac{2}{N-1} e^{-\beta|\delta|}+\cdots\right), & \delta<0, \\
\frac{1}{4}-\frac{1}{N} e^{-\beta \delta(N-1) / 2}+\cdots, & \delta>0,\end{cases} \\
\left\langle S_{f}^{+} S_{g}^{-}\right\rangle & \approx \frac{1}{N-1}\left(\frac{2}{N}\left(\frac{d}{d \beta} \ln Z_{2}-\frac{1}{\beta} \frac{d}{d \delta} \ln Z_{1}\right)-\frac{1}{2}\right) \approx \\
& \approx \begin{cases}\frac{1}{N}\left(\frac{N+1}{4}-\frac{2}{N-1} e^{-\beta|\delta|}+\cdots\right), & \delta<0, \\
\frac{1}{N} e^{-\beta \delta(N-1) / 2}+\cdots, & \delta>0 .\end{cases}
\end{aligned}
$$

Из формул (22) и (24) следует, что корреляционная длина продольных спиновых компонент при низких температурах ведет себя следующим образом: при $\delta>0$

$$
\zeta=\frac{N}{4} e^{\beta \delta(N-1) / 2}
$$

при $\delta<0$

$$
\zeta= \begin{cases}\frac{1}{\ln (N-1)}\left(1-\frac{4}{N \ln (N-1)} e^{-\beta|\delta| / 2}\right), & N \text { четное } \\ \frac{1}{\ln N}\left(1-\frac{8}{(N-1) \ln N} e^{-\beta|\delta|}\right), & N \text { нечетное. }\end{cases}
$$


Таким образом, корреляционная длина при $T \rightarrow 0$ расходится при анизотропии “легкая ось" и остается конечной при анизотропии “легкая плоскость".

Приведем также асимптотические формулы для двухчастичных корреляционных функций и удельной теплоемкости при высоких температурах:

$$
\begin{aligned}
\left\langle S_{f}^{z} S_{g}^{z}\right\rangle & =\frac{\xi}{16} \beta+\frac{1}{64}\left((N-2) \xi^{2}-1\right) \beta^{2}+\cdots \\
\left\langle S_{f}^{+} S_{g}^{-}\right\rangle & =\frac{\beta}{8}+\frac{1}{32}(N-\xi-2) \beta^{2}+\cdots
\end{aligned}
$$

И

$$
\frac{1}{N k_{\mathrm{B}}} C_{V}=\frac{N-1}{32} B^{2} \beta^{2}\left(\xi^{2}+2+\left[(N-2) \xi^{2}-3 \xi+2 N-4\right] \frac{B \beta}{2}+\cdots\right) .
$$

Обсудим вопрос о переходе в рассматриваемой модели к пределу $N \rightarrow \infty$. Очевидно, что в этом предельном случае модель РССВ будет соответствовать модели Гейзенберга с взаимодействием ближайших соседей в бесконечномерном пространстве. В работах [12], [13] было найдено точное решение задачи (1) (при $\xi=1$ ) в термодинамическом пределе $N \rightarrow \infty, V \rightarrow \infty, N / V=$ const, которое совпало с результатами теории молекулярного поля Вейса.

\section{4. ЗАКЛЮЧЕНИЕ}

Проведен точный расчет корреляционных функций и связанных с ними термодинамических характеристик магнитоактивных кластеров на основе модели РСВВ. Разработаны и продемонстрированы два метода расчета корреляционных функций модели. Первый метод основан на непосредственном (по определению) расчете термодинамического среднего, поскольку гамильтониан модели имеет простой вид. Второй метод базируется на решении уравнений движения для спиновых компонент в представлении Гейзенберга и получении на их основе решения алгебраической системы уравнений для искомых корреляционных функций. Этот метод оказывается удобным при расчете корреляционных функций в моделях, в которых затруднено или невозможно непосредственное вычисление термодинамических средних.

Показано, что в нулевом внешнем магнитном поле в кластерах с конечным числом спинов спонтанная намагниченность отсутствует, и это связано с образованием в системе топологических возбуждений (солитонов).

Исследовано поведение теплоемкости, парной корреляционной функции для поперечных и продольных спиновых компонент в зависимости от параметра взаимодействия $\xi$ и числа частиц $N$ в кластере. Найдено, что удельная теплоемкость в нулевом магнитном поле при низких температурах проявляет аномальное поведение, связанное с появлением в системе спиновой щели. Величина и область существования щели существенно определяются типом анизотропии (“легкая ось" или “легкая плоскость"). Для легкоосного спинового кластера спиновая щель значительно шире, чем в легкоплоскостном кластере, а область параметра анизотропии, в которой она открывается, наоборот, уже. Причины образования щели, и, как следствие, аномальное поведение теплоемкости в анизотропном спиновом кластере, 
по-видимому, связано с образованием в системе топологических солитонов как связанных магнонных комплексов, где энергия магнитной анизотропии соответствует парному притяжению длинноволновых магнонов.

Отметим, что мы не учитывали влияние магнитного поля на поведение магнитных и термодинамических свойств модели. Термодинамика модели в присутствии внешнего поля требует дополнительного анализа, результаты которого будут представлены в следующей работе.

Как уже упоминалось в разделе 1, модель РССВ может быть использована для реализации основных операций булевой алгебры в квантовых компьютерах. Отметим еще одно приложение модели РССВ для системы конечных спинов, связанных случайными взаимодействиями. Пусть система $N$ спинов совершает случайные движения вблизи некоторых положений равновесия, и спины взаимодействуют между собой посредством обменных или диполь-дипольных взаимодействий. Если флуктуации, вызванные изменением расстояний между спинами, носят случайный характер, то можно усреднить потенциал взаимодействия по относительным расстояниям $r_{i j}=\mathbf{r}_{i}-\mathbf{r}_{j}, r_{\min }<\left|r_{i j}\right|<r_{\max }$, и перейти к усредненным парным потенциалам и их среднеквадратичным отклонениям:

$$
\begin{gathered}
A=\left\langle U_{i j}^{z z}\right\rangle=\frac{1}{N} \sum_{r_{\min }<\left|r_{i j}\right|<r_{\max }} U^{z z}\left(\left|r_{i j}\right|\right), \\
B=\left\langle U_{i j}^{+-}\right\rangle=\frac{1}{N} \sum_{r_{\min }<\left|r_{i j}\right|<r_{\max }} U^{+-}\left(\left|r_{i j}\right|\right) \\
\Delta A=\left\langle\left(\Delta U_{i j}^{z z}\right)^{2}\right\rangle^{1 / 2}=\left(\frac{1}{N} \sum_{r_{\min }<\left|r_{i j}\right|<r_{\max }}\left(U^{z z}\left(\left|r_{i j}\right|\right)-\left\langle U_{i j}^{z z}\right\rangle\right)^{2}\right)^{1 / 2}, \\
\Delta B=\left\langle\left(\Delta U_{i j}^{+-}\right)^{2}\right\rangle^{1 / 2}=\left(\frac{1}{N} \sum_{r_{\min }<\left|r_{i j}\right|<r_{\max }}\left(U^{+-}\left(\left|r_{i j}\right|\right)-\left\langle U_{i j}^{+-}\right\rangle\right)^{2}\right)^{1 / 2} .
\end{gathered}
$$

Эти выражения расширяют возможности применения модели РССВ и позволяют с единых позиций рассчитать квантовый спектр такой системы и рассмотреть ее термодинамику, пользуясь методом, предложенным в настоящей работе.

ПРИЛОЖЕНИЕ

Рассмотрим альтернативный подход к расчету корреляционных функций $R_{k l}$. В работе [1] были найдены следующие временные зависимости для операторов $S^{ \pm}$:

$$
S^{ \pm}(t)=e^{i t \theta_{ \pm}} S^{ \pm}, \quad \theta_{ \pm}=B(\xi-1)\left(\frac{1}{2} \mp S^{z}\right) .
$$

Воспользуемся тождеством

$$
\left\langle A_{1}(-i \beta) A_{2}\right\rangle=\left\langle A_{2} A_{1}\right\rangle
$$

где $A_{1}(t)$ - некоторый квантово-механический оператор в представлении Гейзенберга, $A_{2}$ - этот же оператор в представлении Шредингера. Данное тождество несет 
главную нагрузку в предлагаемом ниже подходе к расчету корреляционных функций. На его основе мы, пользуясь решениями уравнений движения для операторов изучаемой системы в представлении Гейзенберга, можем свести задачу расчета корреляционных функций к решению системы алгебраических уравнений и тем самым обойти необходимость вычисления статистической суммы задачи. Очевидно, что указанный метод весьма удобен при расчете термодинамических характеристик систем, в которых прямой расчет статистической суммы невозможен или крайне сложен. Отметим также, что использование тождества (П.1) дает возможность непосредственно перейти к задаче расчета корреляционных функций без применения техники метода двухвременных функций Грина, имеющей, как выяснилось в работе [14], ряд недостатков. Подставляем в тождество (П.1) операторы $A_{1}(t)=\left(S^{2}\right)^{k-1}\left(S^{z}\right)^{l-1} S^{+}(t)$ и $A_{2}=S^{-}$, получаем

$$
\left\langle\left(S^{2}\right)^{k-1}\left(S^{z}\right)^{l-1} e^{\beta \theta_{+}} S^{+} S^{-}\right\rangle=\left\langle S^{-}\left(S^{2}\right)^{k-1}\left(S^{z}\right)^{l-1} S^{+}\right\rangle=\left\langle S^{-} S^{+}\left(S^{2}\right)^{k-1}\left(S^{z}+1\right)^{l-1}\right\rangle \text {. }
$$

Исключая операторы $S^{+} S^{-}$и $S^{-} S^{+}$по формуле (2), имеем

$$
\left\langle\left(S^{2}\right)^{k-1}\left(S^{z}\right)^{l-1} e^{\beta \theta_{+}}\left(S^{2}+S^{z}-\left(S^{z}\right)^{2}\right)\right\rangle=\left\langle\left(S^{2}-S^{z}-\left(S^{z}\right)^{2}\right)\left(S^{2}\right)^{k-1}\left(S^{z}+1\right)^{l-1}\right\rangle .
$$

Представим последнее уравнение в виде

$$
\begin{aligned}
\left\langle\left(S^{2}\right)^{k-1}\right. & \left.\left(S^{2} \varphi_{l}(\beta)+\varphi_{l+1}(\beta)-\varphi_{l+2}(\beta)\right)\right\rangle= \\
& =\sum_{p=1}^{l}\left(\begin{array}{l}
l-1 \\
p-1
\end{array}\right)\left\langle\left(S^{2}\right)^{k-1}\left(S^{2} \varphi_{p}(0)-\varphi_{p+1}(0)-\varphi_{p+2}(0)\right)\right\rangle
\end{aligned}
$$

где мы ввели функцию $\varphi_{l}(\beta)=\left(S^{z}\right)^{l-1} e^{\beta \theta_{+}}$, а также воспользовались тождеством

$$
\left(S^{z}+1\right)^{l-1}=\sum_{p=1}^{l}\left(\begin{array}{l}
l-1 \\
p-1
\end{array}\right)\left(S^{z}\right)^{p-1} .
$$

Поскольку оператор $S^{z}$ удовлетворяет тождеству (3), функцию $\varphi_{l}(\beta)$ можно представить в виде многочлена [15]:

$$
\varphi_{l}(\beta)=\sum_{k=1}^{N+1} c_{l k}(\beta)\left(S^{z}\right)^{k-1}
$$

где коэффициенты $c_{k l}(\beta)$ определяются из системы линейных алгебраических уравнений

$$
m^{l-1} e^{\beta B(\xi-1)(1 / 2-m)}=\sum_{k=1}^{N+1} c_{k l}(\beta) m^{k-1}, \quad m=-\frac{N}{2}, \ldots, \frac{N}{2} .
$$

Подставляя в уравнение (П.2) результат из (П.3), представим уравнение (П.2) в виде

$$
\begin{aligned}
& \sum_{p=1}^{N+1}\left\langle c_{l p}(\beta) R_{p, k+1}+\left(c_{l+1, p}(\beta)-c_{l+2, p}(\beta)\right) R_{p, k}\right\rangle= \\
& =\sum_{p, q=1}^{N+1}\left(\begin{array}{l}
l-1 \\
p-1
\end{array}\right) \chi(l-p)\left\langle c_{l p}(0) R_{p, k+1}-\left(c_{l+1, p}(0)+c_{l+2, p}(0)\right) R_{p, k}\right\rangle,
\end{aligned}
$$

где $\chi(m)-$ функция Хевисайда. 
Введем в рассмотрение матрицу

$$
C_{\delta}(\beta)=\left\{c_{l+\delta, k}(\beta) ; l, k=1,2, \ldots, N+1\right\} .
$$

Тогда система уравнений (П.4) перепишется в матричном виде

$$
R_{k+1}=L R_{k}
$$

где

$$
\begin{aligned}
L & =\left[C_{0}(\beta)-V C_{0}(0)\right]^{-1}\left[C_{2}(\beta)-C_{1}(\beta)-V\left(C_{1}(0)+C_{2}(0)\right)\right] \\
V & =\left(\left(\begin{array}{l}
l-1 \\
p-1
\end{array}\right) \chi(l-p) ; l, p=1,2, \ldots, N+1\right), \quad R_{k}=\left\{R_{1 k}, \ldots, R_{N+1, k}\right\} .
\end{aligned}
$$

Решение матричного уравнения (П.5) имеет вид

$$
R_{k}=L^{k-1} R_{1}
$$

и связывает между собой корреляционные функции $R_{k, 1}$ и $R_{k, l}$ при $l>1$. Для определения корреляционных функций $R_{k, 1}$ необходимо получить еще одну систему уравнений. Воспользуемся уравнением, выведенным в статье [1] на основе точного решения уравнения движения для оператора $S_{f}^{z}$ :

$$
\begin{gathered}
\left\langle\left(\Phi_{1}\left(S^{2}, \beta\right) S^{z}-\Phi_{2}\left(S^{2}, \beta\right)\right) S^{+} S_{f}^{-} A\right\rangle-\left\langle\left(\Phi_{1}\left(S^{2}, \beta\right) S^{z}+\Phi_{2}\left(S^{2}, \beta\right)\right) S^{-} S_{f}^{+} A\right\rangle= \\
=\left\langle\left(\Phi_{1}\left(S^{2}, \beta\right)\left(S^{2}-\left(S^{z}\right)^{2}\right)-\Phi_{2}\left(S^{2}, \beta\right)\right) A\right\rangle
\end{gathered}
$$

где

$$
\begin{aligned}
& \Phi_{1}\left(S^{2}, \beta\right)=\frac{e^{\beta / 2}(\operatorname{sh}(\beta \Omega)-2 \Omega \operatorname{ch}(\beta \Omega))+2 \Omega}{2 \Omega S^{2}} \\
& \Phi_{2}\left(S^{2}, \beta\right)=\frac{e^{\beta / 2} \operatorname{sh}(\beta \Omega)}{\Omega}, \quad \Omega=\sqrt{S^{2}+\frac{1}{4}}
\end{aligned}
$$

Просуммировав равенства (П.7) по $f$ и воспользовавшись коммутационным соотношением для операторов $S^{ \pm}$, а также выражением (2) для $S^{2}$, получим

$$
\left\langle F_{1}\left(S^{2}, \beta\right)\left(S^{z}\right)^{2} A\right\rangle=\left\langle F_{2}\left(S^{2}, \beta\right) A\right\rangle,
$$

где

$$
\begin{aligned}
& F_{1}\left(S^{2}, \beta\right)=\left(\frac{N}{2}+1\right) \Phi_{1}\left(S^{2}, \beta\right)+\Phi_{2}\left(S^{2}, \beta\right) \\
& F_{2}\left(S^{2}, \beta\right)=\frac{N}{2} S^{2} \Phi_{1}\left(S^{2}, \beta\right)+\left(S^{2}-\frac{N}{2}\right) \Phi_{2}\left(S^{2}, \beta\right) .
\end{aligned}
$$

Так как оператор $S^{2}$ удовлетворяет тождеству (4), операторные функции $F_{1,2}\left(S^{2}, \beta\right)$ можно представить как многочлены [15]:

$$
F_{1,2}\left(S^{2}, \beta\right)=\sum_{k=1}^{[N / 2]+1} \xi_{k}^{1,2}\left(S^{2}\right)^{k-1},
$$


где коэффициенты $\xi_{k}^{1,2}$ определяются из системы уравнений

$$
F_{1,2}(s(s+1), \beta)=\sum_{k=1}^{[N / 2]+1} \xi_{k}^{1,2}(s(s+1))^{k-1}, \quad s=s_{\min }, \ldots, \frac{N}{2} .
$$

Используя тождество (П.9) и полагая $A=\left(S^{z}\right)^{p-1}$, перепишем уравнение (П.8) в виде

$$
\sum_{k=1}^{[N / 2]+1} \xi_{k}^{1}\left\langle\left(S^{2}\right)^{k-1}\left(S^{z}\right)^{p+2-1}\right\rangle=\sum_{k=1}^{[N / 2]+1} \xi_{k}^{2}\left\langle\left(S^{2}\right)^{k-1}\left(S^{z}\right)^{p-1}\right\rangle .
$$

Поскольку $R_{11}=1$, данная система уравнений принимает вид

$$
W_{p 1}+\sum_{q=2}^{N+2} W_{p q} R_{q 1}=0
$$

где матрица $W$ определяется равенством

$$
W=\sum_{k=1}^{[N / 2]+1}\left(\xi_{k}^{1} C_{2}(0)-\xi_{k}^{2} E\right) L^{k-1} .
$$

Система уравнений (П.10) определяет искомые корреляционные функции $R_{q 1}$, зная которые, нетрудно найти остальные корреляционные функции $R_{p q}$, воспользовавшись уравнением (П.6).

Расчет корреляционных функций $R_{k l}$ по формулам (П.6) и (П.10) приводит к результату (6).

\section{Список литературы}

[1] А.Р. Кессель, Р. Р. Нигматуллин, А. А. Хамзин, Н. А. Яковлева, ТМФ, 145:3 (2005), 411-419.

[2] A. K. Khitrin, V.L. Ermakov, B. M. Fung, Chem. Phys. Lett., 360:1-2 (2002), 161-166, arXiv: quant-ph/0202035.

[3] A. K. Khitrin, V. L. Ermakov, B. M. Fung, J. Chem. Phys., 117:15 (2002), 6903-6906.

[4] М. Г. Рудавец, Э. Б. Фельдман, Писъма в ЖЖЭФ, 75:12 (2002), 760-762.

[5] А. О. Гладкий, А. В. Любезный, О. В. Черановский, Вестн. Харък. нац. ун-та. Сер. химия, 731:14(37) (2006), 30-36.

[6] М. А. Юрищев, ЖЭТФ, 131:3 (2007), 511-517.

[7] O. G. Mouritsen, H. Jensen, H. C. Fogedby, Phys. Rev. B, 30:1 (1984), 498-500.

[8] Ю. А. Изюмов, УФН, 155:4 (1988), 553-592.

[9] A. P. Ramirez, W. P. Wolf, Phys. Rev. Lett., 49:3 (1982), 227-230.

[10] Б. А. Иванов, А. М. Косевич, Писъма в ЖЭТФ, 24:9 (1976), 495-499.

[11] А. С. Ковалев, А. М. Косевич, К. В. Маслов, Писъма в ЖЭЭФ, 30:6 (1979), 321-324.

[12] G. Scharf, Phys. Lett. A, 38:2 (1972), 123-124.

[13] Б. В. Мощинский, В. К. Федянин, ТМФ, 31:1 (1977), 101-106.

[14] М. Ф. Сарры, УФН, 161:11 (1991), 47-92.

[15] Ф.Р. Гантмахер, Теория матрии, Наука, М., 1988.

Поступила в редакцию 28.10.2009, после доработки 1.04.2010 